\title{
Colored video analysis in wireless capsule endoscopy: a survey of state-of-the-art
}

Article

Accepted Version

Ashour, A. S., Dey, N., Mohamed, W. S., Tromp, J. G., Sherratt, S., Shi, F. and Luminița, M. (2020) Colored video analysis in wireless capsule endoscopy: a survey of state-ofthe-art. Current Medical Imaging, 16 (9). pp. 1074-1084. ISSN 1875-6603 doi:

https://doi.org/10.2174/1573405616666200124140915 Available at https://centaur.reading.ac.uk/88629/

It is advisable to refer to the publisher's version if you intend to cite from the work. See Guidance on citing.

To link to this article DOI:

http://dx.doi.org/10.2174/1573405616666200124140915

Publisher: Bentham Science

All outputs in CentAUR are protected by Intellectual Property Rights law, including copyright law. Copyright and IPR is retained by the creators or other copyright holders. Terms and conditions for use of this material are defined in the End User Agreement.

\section{www.reading.ac.uk/centaur}


Central Archive at the University of Reading

Reading's research outputs online 


\title{
Colored Video Analysis in Wireless Capsule Endoscopy: A Survey of State-of-the-Art
}

\author{
Amira S. Ashour ${ }^{* a}$, Nilanjan Dey ${ }^{\mathrm{b}}$, Waleed S. Mohamed ${ }^{\mathrm{c}}$, Jolanda G. Tromp ${ }^{\mathrm{d}}$, R. Simon Sherratt ${ }^{\mathrm{e}}$, \\ Fuqian Shi ${ }^{\mathrm{f}}$ and Luminița Moraru ${ }^{\mathrm{g}}$
}

\author{
${ }^{a}$ Department of Electronics and Electrical Communications Engineering, Faculty of Engineering, Tanta University, \\ Tanta, 31527, Egypt; \\ ${ }^{b}$ Department of Information Technology, Techno India College of Technology, West Bengal, 740000, India; \\ ${ }^{c}$ Department of Internal Medicine, Faculty of Medicine, Tanta University, Tanta, 31527, Egypt; \\ ${ }^{\mathrm{d} D i r e c t o r}$ of the Center for Visualization and Simulation, Computer Science Department, Duy Tan University, Da Nang, \\ Vietnam; \\ 'Department of Biomedical Engineering, the University of Reading, RG6 6AY, UK; \\ ${ }^{f}$ Wenzhou Medical University, Wenzhou, 325035, P.R. China; \\ ${ }^{g}$ Faculty of Sciences and Environment, Dunarea de Jos University of Galati, 47 Domneasca St., 800008, Romania
}

*Corresponding author: (email: amirasashour@yahoo.com, amira.salah@f-eng.tanta.edu.eg)

\begin{abstract}
:
Background: Wireless Capsule Endoscopy (WCE) is a highly promising technology for gastrointestinal (GI) tract abnormality diagnosis. However, low image resolution and low frame rates are challenging issues in WCE. In addition, the relevant frames containing the features of interest for accurate diagnosis only constitute $1 \%$ of the complete video information. For these reasons, analyzing the WCE videos is still a time consuming and laborious examination for the gastroenterologists, which reduces WCE system usability. This leads to the emergent need to speed-up and automates the WCE video process for GI tract examinations.

Objective: Consequently, the present work introduced the concept of WCE technology, including the structure of WCE systems, with a focus on the medical endoscopy video capturing process using image sensors. It discussed also the significant characteristics of the different GI tract for effective feature extraction. Furthermore, video approaches for bleeding and lesion detection in the WCE video were reported with computer-aided diagnosis systems in different applications to support the gastroenterologist in the WCE video analysis. Conclusion: In image enhancement, WCE video review time reduction is also discussed, while reporting the challenges and future perspectives, including the new trend to employ the deep learning models for feature Learning, polyp recognition, and classification, as a new opportunity for researchers to develop future WCE video analysis techniques.
\end{abstract}

Keywords: Endoscopy capsule, video analysis, bleeding detection, reviewing time reduction, wireless video gastrointestinal (GI) endoscopy capsule, computer- aided diagnosis.

\section{INTRODUCTION}

Colon cancers and gastrointestinal $(\mathrm{GI})$ inflammation/disorders are amongst the most chronic gastrointestinal tract system problems. Early-stage detection of most GI cancers can contribute to surviving such serious diseases. Several generic analysis methods, including double-contrast barium enema and Computed Tomography (CT) can be used for detecting the GI tract disease. Conversely, the traditional endoscopy facilitates accurate diagnosis, founded on biopsy samples and real video analysis [1]. This directs the researchers' efforts toward improving the endoscope, leading to the noninvasive realistic Wireless Capsule Endoscopy (WCE), which has revolutionized diagnostic technology by detecting the whole GI tract $[2,3]$. The WCE was developed to monitor the whole GI tract, particularly, the small bowel to impact the diagnostic methodology for several diseases, including celiac and Crohn's diseases, bleeding, polyps and cancers, and other lesions [3].

Commonly, the WCE travels forward into the sink (i.e. the end of the GI tract); however, sometimes it may travel backward. In addition, the transit time of the WCE is variable and can extend to 8 hours. The WCE can speedily gain recognition within the GI tract due to its two key advantages, namely i) it offers the entire small intestine inner visualization, and ii) it acquires the images in a non-invasive 
way to decrease the patient's examination time. During the WCE path through the GI tract, it records video which consequently requires time-consuming photographic assessment [4]. The assessment encompasses the video frame inspection, which requires WCE footage analysis to reduce the diagnosis time and the procedure cost by using a computer-aided diagnosis (CAD) system.

Additionally, the WCE procedure has several limitations [5], such as i) the huge required analysis time for the whole video, where the WCE releases, images at a rate of two frames/second terminated after about eight hours resulting in about 57,600 images for a patient, ii) the WCE has no therapeutic ability, thus if any injury is exposed, other extra tests will be essential, and iii) the complexity to discern the precise visualized lesion location. Unfortunately, the WCE has limited operating time, low image resolution and low frame rate compared with the wired endoscopy, which is considered the WCE bottleneck. Thus, the WCE video analysis automation has a dramatic effect on the capsule importance and the diagnosis cost/ accuracy along with increasing the image contrast. Such analysis requires image processing and computer vision processes, including i) the WCE video segmentation into significant parts, namely the mouth, oesophagus, stomach, small intestine and colon, ii) features extraction from the captured images in the video based on texture, color and shape and bleeding detection, and iii) classification into several target classes, such as normal, polyps, lesions and cancer. The main task of such image processing techniques is to visualize and to support the WCE video. Several software tools have been developed to interface and to analyze the WCE data. Consequently, the present work outlines the WCE technology progress along with the development tendencies of the required video analysis to acquire high frame rate, a high image resolution, and less operational time.

The organization of the following sections is as follows, the technology of the WCE is introduced in section 2 followed by the feature extraction process from WCE images and WCE video segmentation and classification in sections 3 and 4 , respectively. Section 5 highlights the WCE video visualization and compaction followed by WCE video processing in section 6 . In section 7 , the WCE based computer-aided diagnosis systems were introduced. In section 8, research directions and challenges are reported followed by the conclusion in section 9 .

\section{WIRELESS CAPSULE ENDOSCOPY VIDEO TECHNOLOGY}

In recent years, WCE becomes an adopted clinical routine in the medical domain due to the development of CAD systems [6]. Compared to conventional endoscopy, WCE has a wider flexible range of view to inspect larger areas of the intestine as illustrated in Fig. 1.

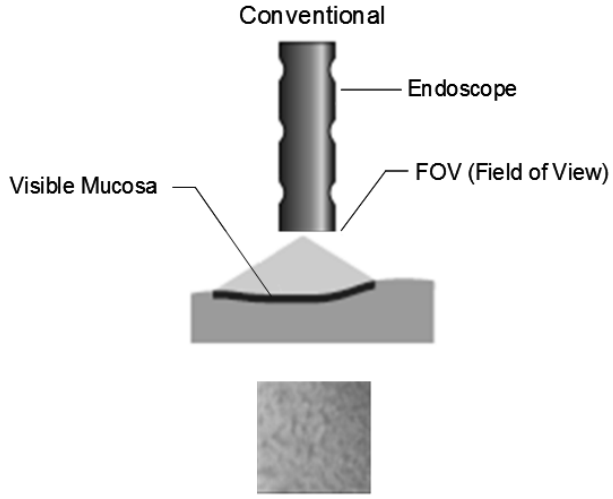

(a)

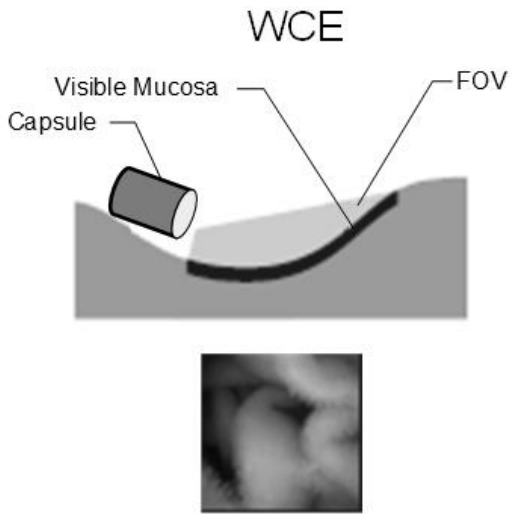

(b)

Fig. 1. (a) Conventional endoscopic imaging, (b) WCE

The WCE systems were deliberated for abnormality detection and/or classification to provide accurate medical diagnosis [7]. This non-invasive clinical technique allows the entire GI tract to be inspected using a small encapsulated CMOS (Complementary Metal-Oxide Semiconductor) camera. This system was established in 2000 [8] leading to the first marketable system 'M2A' from Given Imaging Ltd. that followed the American Food and Drug Administration (FDA) clearance. The 'M2A' system is represented as 'PillCam SB', which includes a small $(11 \mathrm{~mm} \times 26 \mathrm{~mm})$ capsule that is linked with a data-recorder belt. During the investigation process, the capsule is swallowed and driven down the GI tract by peristalsis before being naturally expelled. For illumination, one end of the capsule has a transparent optical dome containing six LEDs (Light Emitting Diodes). The LED array surrounds the camera used to capture two-color images per second. The images are JPEG (Joint Photographic Experts Group) compressed and transferred using radiotelemetry to the worn data recorder on the patient's belt. Afterward, the received Radio Frequency (RF) signal by the fixed antenna array on the body of the patient is analyzed using software tools in order to determine the capsule location and trajectory [9].

In 2004, a second version called 'PillCam ESO' was released by Given Imaging, which included two CMOS cameras functioning at a higher frame rate designed to target oesophageal disease. In 2006, another 'PillCam COLON' capsule was created for colon inspection. Later in 2005, the 'EndoCapsule' system was launched by Olympus using a CCD (Charged Coupled Device) camera system accompanied by $\mathrm{ABC}$ (Automatic Brightness Control) for 
providing programmed illumination adjustment to guarantee images of higher resolution with consistent quality. This was followed by the 'PillCam SB2' capsule in 2007.

\subsection{Endoscopy capsule video capturing using image sensors}

Recently, endoscopes have combined minute video cameras to deliver maximum flexibility. The small diameter endoscope probe tip defines the light source, space shared by the camera, a biopsy/suction channel and the air/ water nozzle. Using a CMOS fabrication process, such as Forza Silicon, produces highly-miniaturized image sensors, where Forza is able to create designs that meet the increasing surgical demands, such as higher resolution. Imaging abilities, including the illumination as well as the sensor characteristics are considered the most significant features in designing WCE systems [10]. Typically, the temporal resolution defines the information covered by the capsule through its path in the patient's body. Additionally, the spatial resolution defines the diagnostic quality that can be attained for site analysis. The position of the sensor/ lens determines the region imaged by the WCE. Most of the image sensors are attached to the capsule. Lenticular lens arrays or microlens arrays are established in laparoscopic surgery to deliver a multi-view image by a single sensor. Generally, the capsules should offer a widespread Field of View (FoV) to detect an adequate tissue wall image [11]. The image quality is intrinsically directed by the sensor abilities and illumination of the WCE. Several illumination strategies can be engaged to conserve power. The brightness of the overall image can be further modified to preserve the image quality to aid investigation. The most known signal modality for exploration by the physicians in WCE imaging is the white light.

The Autofluorescence capsule [11] may be used for disease detection without an on-board camera. Data transmission and reception is a prevalent issue in WCE equipment. In order to enable high-resolution imaging, a high data rate scheme is indispensable. In the capsule design, the telemetry subsystem forms a bottleneck due to the size constraint limitations of the wireless communication system. Furthermore, videobased motion tracking is a critical issue, where at least one camera is used to equip the WCE to offer visual inspection of the GI tract and to track the capsule movement. Inconsistent with Shannon's information entropy, the more correlation two images have more mutual information. Thus, the iterative change of the scaling and rotation coefficients can be used to acquire the rotation and displacement angle.

\subsection{Wireless capsule endoscopy system}

The wireless video GI endoscopy capsule is considered as a micro-device that empowers the invasive investigation of the human body. Generally, the GI tract examination has the following procedure. Initially, the WCE is swallowed by the patient and starts capturing images through its movement in the GI tract. The WCE transmits compressed images to be received outside the body. One or two miniature cameras are attached to the WCE to capture thousands of images through the capsule path in the GI tract. The capture rate is currently about two frames/ second through its movement in the GI tract, including the stomach, esophagus, and small/large intestines. The captured still images from a pseudo video sequence [12]. Finally, the WCE is reflexively ejected from the human body. However, this effective method suffers from relatively high power consumption of the camera and RF components, which leads to a limited 8-12 hours WCE lifetime to examine the small intestine and large intestine. Increasing functionality is a critical requirement for endoscopic system platforms, which are considered HighDefinition (HD) systems. The image sensor and processing interpolation/engine systems are significant to achieve effective resolution. The WCE system has some features, including multiple camera feeds, side-by-side, viewing, picture-in-picture, and multiple image viewing [9]. Radiographic and archived images may be presented alongside with the endoscopic video feed for comparison. Video transport to the video monitors requires support for innumerable interface standards, including CameraLink, DisplayPort, GigE Vision, HDMI, SDI and HD-SDI. However, further enhancements are required to guarantee effective surgical room usage with shorter set-up time, increased optical zoom capabilities, depth of focus, light intensity, and better digital zoom. Typically, the WCE system equipment necessitates increased processing performance, where image sensor technology develops higher resolution streams [13]. The physical endoscopy system equipment is demonstrated in Figure 2.

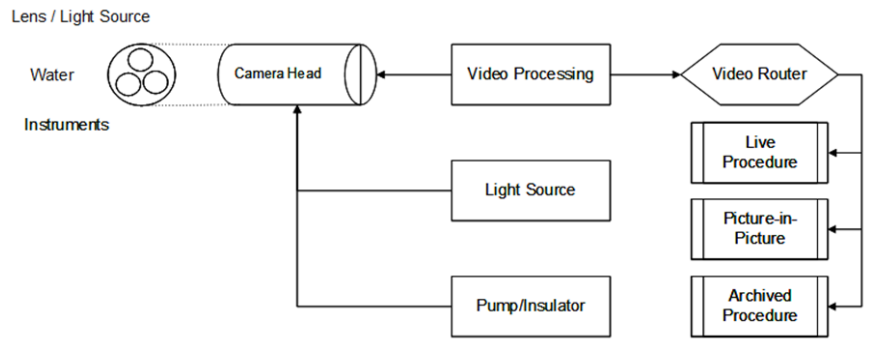

Fig. 2. Endoscopic system diagram

The endoscopic system contains several components, including hand controls that enclose the light path, lens, water/gas outlets and other tools. The endoscope head is connected to a light source through fiber optics to control high-intensity illumination. A camera head containing the image sensor (single or three-chip arrays) as well as video pre-processing electronics and a video recorder is also connected to the endoscope. Finally, insulators for injecting $\mathrm{CO} 2$ into the body cavities also exist in the endoscope to preserve the pressure level in the body in order to view the tissues and organs within the body. The endoscopic system has enabled physicians to diagnose the GI pathologies in the colon, duodenum, and stomach. However, the examination of the small intestine using the traditional endoscopy has been limited.

\section{WCE IMAGE FEATURES EXTRACTION}

Once the WCE images are received, they are available to be analyzed and processed by the CAD to convert the visible information into different features based on the tackled problem. During the WCE operation, monotonic/slow movement and low resolution due to the passive force of the camera via the intestine triggered by the peristalsis characterize the captured data. Due to the WCE data nature, spatio-temporal features are a promising concept. With the intention of extracting these features, shape fitting, edge detection, or segmentation algorithms are carried out [14]. 
These features include color-based features using the color histogram, textural features, such as Local Binary Pattern (LBP) [15], bag-of-visual-words features using SIFT (Scaleinvariant feature transform) [16], gradient-based features using Histogram of Gradients (HoG) [17], and blob detectors using Laplacian of Gaussian (LoG) as well as using higherlevel features in certain situations to define the image.

Generally, in the WCE video, each part of the digestive system has its significant color, such as the mouth color is unsaturated, the stomach color is pink; the small intestine and the colon have pink to yellow colors, while due to faecal contamination, the colon also may often be blocked by varying yellow to green colors. Furthermore, different color signatures can be signified dissimilar pathologies, such as yellow color can refer to ulcerations and white colors enclosed by excessively reddish refers to bleeding or inflammation. Thus, for object recognition and image analysis in the WCE real colored video, the color distribution plays a significant role, which is represented using the color histogram approach. This approach is considered invariant to rotation/translation about the viewing axis, partial occlusion and image scale changes. Typically, the WCE video frames are transmitted and deposited as RGB (Red-Green-Blue) triplets for further color feature extraction. However, Berens et al. [18], Berens and Fisher [19], Gong et al. [20] and Manjunath et al. [21] all established that using the HSI (Hue, Saturation, Intensity) color space produced a superior classification compared to RGB. Moreover, the variation in the distance between the intestinal surface and the capsule leads to the intensity dissimilarity in the WCE images, thus, the HS histograms have been applied [19]. Moreover, Seguí et al. [22] recommended and implemented a deep learning model for extracting generic feature descriptor from the WCE images.

Furthermore, texture features have an imperative role in the segmentation of WCE video. The different parts of the digestive system can be distinguished using the 'villi' texture pattern, which are small finger-like projections responsible for food absorption. These patterns exist in the small intestine, but do not exist in the colon and stomach neighboring regions. The 3D-LBP operator has been introduced [23], which independently has been shown to calculate 1D-LBP histograms for RGB color channels. Moreover, air bubbles, strong shadows, and other artifacts, including the bile, mucus, food, and faeces can obscure WCE images, which lead to visual contamination affecting the histogram analysis. This problem can be overcome by extracting only the image parts that contain non-occluded tissues [24].

\section{WCE VIDEO SEGMENTATION CLASSIFICATION}

AND

The GI tract in the digestive system includes several parts, namely the rectum, colon, ileum, jejunum, duodenum, stomach, oesophagus, and mouth. Each part is considered a Region of Interest (ROI) during the video analysis process, which requires segmentation processing to identify and to localize each part accurately. In addition to detecting the blood and the bleeding regions within the GI tract, if they exist, for further diagnosis.

\subsection{Topographic video detection}

Determining the capsule path using WCE video segmentation is considered to be a video segmentation process to segment the capsule video into evocative GI tract parts. This requires the proper selection of the significant related features of the part under concern. The starting point of the capsule journey takes limited time to pass via the oesophagus before entering the stomach, where it typically remains from 15 minutes to several hours, until it passes out through the pylorus valve. Typically, the pylorus localization in a video is a time consuming and complex process, where the tissues of the stomach are near to the pyloric and the small intestine has similar visualization. Afterwards, in order to reach the colon, the WCE takes about four hours to transit the small intestine. In addition, the colon entry is difficult because of the ileocaecal valve, as the tissue is often obscured by faecal material.

The most popular GI tract actual videos segmented distinctive anatomical regions include the oesophagus; mouth; small intestine; stomach and colon. Lee et al. [25] segmented the WCE video into the digestive organs based on their different intestinal contraction patterns. Frequency functions analysis related to these patterns has been carried out to determine the boundaries of the consecutive organ entrance.

\subsection{Detection of bleeding, lesion and GI tract organs}

Blood and bleeding detection in the GI tract is considered a significant issue in WCE that has been considered by several studies. Detection of the WCE GI bleeding is essential for examination as it is considered the most common GI tract abnormality as well as it is an imperative syndrome/symptom of other GI pathologies including polyps, ulcers, tumors and Crohn's disease. The primary effort in WCE-CAD systems have been directed to bleeding and lesion detection. There are two main categories for GI bleeding, namely the active bleeding and the inactive bleeding that may occur anywhere throughout the GI tract [26]. For clarifying the WCE video's results of the review procedure for bleeding detection, the following types of features and processes are considered in the image processing techniques. These features include i) texturebased features that consider the spatial associations between pixels, ii) the colour-based methods that handle the image as sets of the individual values are applied, and iii) contourbased methods that focus on contour and edge detection. Finally, a decision can be taken after interpreting the regions of interest for decision about the bleeding presence.

Usually, bleeding is detected by the color analysis commonly in HSI space. After segmentation, feature extraction and the bleeding detection process are performed for further classification process using different algorithms, such as the neural network [27-30], support vector machines (SVM) [31] and conventional neural networks [32]. Consequently, to collect the training, validation and testing sets for WCE, researchers have been attempting to resolve the associated intrinsic shortcomings of the video screening phase, namely the required long visualization time.

For bleeding detection, it has been established that color information can be adequate; however, some descriptors ought to be employed for lesion detection. Several approaches have been offered for polyp detection, such as 
curvature information to obtain polyp segments [33], Gabor filters based segmentation improved using an edge detector [34], and texture information and integrate color using LBP [35]. New approaches, namely Bayesian Information Criterion (BIC) and Expectation Maximization (EM) clustering have been presented to detect the bleeding areas in the WCE video [36]. Through this approach, the images have been segmented into non- bleeding and bleeding regions for further modeling of these regions' color distribution by applying Gaussian mixtures in RGB color space, finally calculating the bleeding region areas. In order to evaluate this parametric bleeding color distribution model, 15,222 WCE images of which 1,731 contained bleeding regions have been tested from three different videos. The results reported sensitivity and specificity of $92.55 \%$ and $98.10 \%$, respectively. Moreover, Jung et al. [37] detected the bleeding regions in WCE frames using a color spectrum transformation with threshold. Lately, the chromaticity moments constructed from the Tchebichef polynomials have been used by Li and Meng [38] to detect ulceration and bleeding. WCE images have been divided into thirty-six non-overlapping grids of $30 \times 30$ pixel blocks to calculate six chromaticity moments. Afterwards, from ten patients' video sequences containing 1800 normal-, 1800 ulceration- and 1800 bleeding- image's cases, image blocks were selected from 300 non-consecutive WCE images. The blocks were randomized and classified using a Multi-Layer Perceptron (MLP) neural network.

Furthermore, wavelet based LBP have been used to detect tumors [39]. In order to detect ulcers, the Bag-of-Words (BoW) method based on SIFT features and LBP has been proposed [40]. Furthermore, for polyp detection in WCE images, a stacked sparse autoencoder with image manifold constraint based deep feature learning technique has been implemented [41]. Moreover, in order to detect Crohn's disease, the MPEG-7 descriptors for texture, color, and edge have been used [42]. Several studies have been conducted to classify the bleeding cases. Li and Meng [38] proposed the chromaticity moment along with a NN classifier. Additionally, in WCE images, Sekuboyinaet al. [43] designed a convolutional neural network $(\mathrm{CNN})$ model for abnormality detection, where the images were split into different patches for further features extraction to each block using the proposed CNN.

Another direction of classification based CAD systems is focused on classifying the diverse intestinal tract organs, such as the duodenum, esophagus, jejunum-ileum, stomach and cecum. Igual et al. [44] used the color change pattern to detect the unlike organs in the GI tract. Cunha et al. [45] estimated the locations of the ileo-cecal valve, pylorus and the esogastric junction using MPEG-7 visual descriptors. Recently, Zou et al. classified the digestive organs in the WCE images using a deep convolutional neural network [44]. Generally, for supporting the WCE video processing during the WCE classification process to develop a CAD system, training sets in WCE become essential. Several studies have been carried out on intestinal content, lesions, or event detection using visually ground-truth data sets recognized by clinical experts [38]. The WCE data collection is considered a challenging task, where the training and testing of the dataset size is rather small. This has complicated the development of advanced CAD systems for WCE applications due to such a small amount of available data. As reported by Vilariño et al. [46], the majority of WCE studies have used less than ten video cases to accomplish the performance of the proposed algorithms.

\section{WCE VIDEO VISUALIZATION COMPACTION}

AND

Recently, researchers are motivated to develop video compression techniques for the WCE video visualization to eliminate/ compact similar frames and to apply variable sampling rates at the acquisition step [47]. In order to reduce the scanning process and, hence, the required time for video visualization, the compaction process essential to eliminate the WCE video frames at which the capsule and the intestine are paralyzed.

In order to estimate the capsule and the intestine motion between two successive frames, a deformable ring model was proposed by Szczypiński et al. [48]. Automatic controls of the WCE display rates have been performed to estimate the color distribution change between two consecutive frames. Non-informative frame detection has been addressed to detect the intestinal content in several studies. In order to detect the intestinal content, the color histograms along with the SVM classifier has been used [49]. The intestinal juices' bubble-like shape has been detected by Vilariño et al. [50] based on Gabor filters. A three-stage cascaded technique has been proposed [51] to detect informative frames, where i) the color information is used to distinguish the turbid by applying color moments and histogram with SVM classifier, ii) texture segmentation using Gauss Laguerre Transform has been applied to illustrate the bubbles, and iii) a final threshold on the segmented regions has been used to detect the informative frames. In WCE videos of small intestine, Wang et al. [52] proposed a color-saliency region detection technique to extract the saliency region of interest for protruding lesion detection.

Other interesting directions for the WCE video visualization, focus on the intestinal motility characterization and detection at specific occasions, such as the wrinkles and contractions detection [53]. A consecutive process has been proposed [48] based on i) the gray level image co-occurrence matrix and the LBP for textural features, ii) the mean lumen color for color feature extraction and iii) the LoG filter to extract the blob features. The extracted features information is mined for nine consecutive frames for further classification using the SVM classifier. In $\mathrm{Vu}$ et al. [54], a three-stage process was proposed for the WCE video using the following stages, i) an edge detector has been applied to find the WCE video sequences with possible contractions, ii) the match between the frames' light-intensity histograms has been assessed to remove the non-contractions and, finally, iii) the existence of wrinkle-like pattern, that has been designated using the edge direction histogram, in the sequence's central frames has been used to detect the real contractions.

The motility evaluation of the WCE images have been estimated [55]. From the WCE videos, several motility descriptors have been extracted, where each descriptor signifies a specific intestinal event, such as the video coverage with intestinal content and the number of contractions. Afterwards, for each video, the extracted characteristics have been combined in a feature vector to 
finally conclude the motility of the small intestine using a non-linear two class SVM classifier.

Other research has been interested in the detection of wrinkle frames. For instance, a structure tensor matrix has been employed to originate an image descriptor [56]. An alternative scheme has been considered [57] using a tonic contractions detector based on wrinkle information, where general linear radial patterns have been used as features. The WCE frame has been divided into four different quadrants located according to the lumen center $[56,57]$. The features have been calculated for each quadrant and classified with the SVM. Other researchers have studied the static sequences and tunnel detection in WCE videos. Typically, the static sequences in the WCE video are described by the color distribution change between successive frames. Tunnel detection can be performed by exploring the lumen change in a successive frame sequence.

\section{WCE VIDEO PROCESSING}

High image contrast/resolution, high frame rate and less operational time are the main challenges of WCE video processing technology.

\subsection{Image quality enhancement}

Video quality enhancement of WCE captured raw images are an application area of image processing that is mainly concerned with noise reduction and image visualization enhancement for spotting the relevant regions more easily. The commercial hardware limitations of the WCE platforms result in quality deterioration of the acquired video. Consequently, several images/ video processing algorithms have been proposed to overcome these limitations [58]. The Olympus EndoView is considered to be the first commercial viewing software that uses texture and contrast enhancement procedures before exhibiting the captured images [59].

The inadequate illumination of the mucosal in the GI tract results in dark regions in the WCE images, thus, contrast enhancement should be non-uniform. Since for abnormalities assessment, color is considered the most relevant feature, contrast enhancement becomes indispensable to reserve the WCE images' color tones [60]. Likewise, contrast enhancement is essential to avoid the resulting amplification of noise from the wireless transmission system of WCE and the image sensor. Consequently, image filtering procedures that are reliant on manual adjustment of the parameters, such as homomorphic [61] and anisotropic [62] can be applied efficiently. In addition, noise suppression can be performed by applying common filtering approaches [63]. Moreover, due to the intestinal content interfering with the camera or the WCE abrupt motion, blurring may be occurring. Thus, image-restoration algorithms are vital to cope with the WCE image de-blurring [63].

Artificially, low and limited WCE video sequence framerates can be increased by using algorithms that introduce more frames with predicted content through an interpolation process [64]. Besides, each video frame resolution can be increased using super-resolution algorithms [65]. These algorithms increase the video frame-rate by combining redundant information in the consecutive video frames leading to increased resolution in new frames.

\subsection{Time reduction in the WCE video process}

Abnormality detection can be considered an indirect methodology to reduce the review time, while one of the computer vision techniques in the WCE video analysis is to reduce the required overall time directly for reviewing the data through alerting physicians to the significant video frames. This adaptive viewing speed enhancement can also be achieved by automatic segmentation of the video into significant parts, event detection, or by modifying the number of displayed frames/second (replay speed). Hai et al. [66] discussed the automatic adjustment of the video frame rate instead of requiring manual adjustment of the frame rate. The proposed approach runs the video at high speed in stable areas and at slower speeds at the substantial variations between frames occur to signify the pathologies possibility.

Commercially, effective and simple software can provide multiple consecutive frames' visualization by simultaneously enabling multiple frame evaluation concurrently to achieve potential time reduction [67]. Nevertheless, this prospective is limited by the physician's perception abilities. Thus, another technique is based on epitomes which are created from consecutive frames set [68]. Another method for reviewing time reduction is the panoramic visualization at which the panoramic images are generated automatically from multiple successive video frames by stitching or comparing together successive WCE frames at their matching points [69]. This procedure is repeated for successive frames' clusters to produce new video containing fewer panoramic frames. This new video contains a smaller frame number compared to the original video and provides a wider FoV.

\section{WCE BASED COMPUTER-AIDED DIAGNOSIS SYSTEMS}

$\mathrm{CAD}$ and decision support systems provide physicians with accurate and fast diagnoses along with increased tolerance to handle incomplete/missing data. Developing intelligent diagnostic systems for the WCE image analysis has emerged. WCE constitutes an interesting technology in which the patients swallow a capsule with its embedded micro-camera. Consequently, in capsule image analysis, computer vision has a significant role, which has four stages. These are i) the WCE video topographic segmentation into expressive parts, namely the stomach, oesophagus, mouth, colon and small intestine, ii) the significant videos of the clinical events detection, such as normal and abnormal cases detection, detection of the intestinal fluids, intestinal contractions, physical abnormality, the capsule retention and the bleeding areas, iii) video analysis regarding successive frame changes to adjust the video viewing speed adaptively and to reduce the visualization time and iv) the quality enhancement of the captured raw images by the WCE using image processing. These categories were discussed in detail in section 6; consequently, this current section introduces the application of the CAD systems, which include the different image/video processing stages of the WCE data.

Bleeding is considered to be one of the most perilous GI tract abnormalities, which is primarily detected by the physicians checking the entire WCE video sequence. However, this manual process is time consuming and extremely laborious. Consequently, the advance of CAD 
systems for automatic GI bleeding detection is highly anticipated. In WCE videos, computerized methods for GI bleeding detection have attracted much attention for relieving the physicians' workload. Jia and Meng [70] developed a CAD system for GI automatic bleeding discovery in WCE image videos using the deep convolutional neural network. The proposed method has been evaluated by a dataset of 10,000 WCE images. The results depicted an increase of around 2\% in the F1-score of up to 0.9955 values. Thus the proposed technique outperformed the state-of-the-art methods in WCE bleeding detection.

For ulcer screening, Li and Meng [71] combined the merits of LBP and the Discrete Curvelet Transform (DCT) as a new texture feature. This led to superior texture descriptions with multi-directional characteristic. Charisis et al. [72] processed the entire WCE images with a color rotation operation followed by uniform rotation invariant LBP to extract the features for ulcer. Furthermore, several CAD systems have been designed for intestinal fluid detection. Typically, the intestinal fluids appear as yellow to brown semi-opaque turbid liquids that contain artifacts and air-bubbles. Vilariño et al. [50] proposed an approach based on texture analysis using Gabor filters, where the most relevant features in intestinal fluids of different sized small bubbles as well as quasi-circular shapes were detected. Frames containing detected bubbles were more than 50\% have been considered invalid for clinician analysis. Ten WCE videos have been conducted to evaluate the proposed method in which the number of frames has been reduced from 12 to $46 \%$.

For abnormality detection in the WCE images, Boulougoura et al. [73] designed an intelligent system for discerning between abnormal and normal tissues using fifty-four feature vector components combining nine statistical measurements, namely the kurtosis, skew, variance, standard deviation, energy, entropy, covariance and inverse difference moment that were calculated from six channel histograms $(\mathrm{H}, \mathrm{S}, \mathrm{V}$ and $R, G, B)$. Afterwards, the images were classified using neural networks, leading to $100 \%$ detection accuracy when evaluated by 73 images from the WCE video. In order to detect the intestinal contractions in a WCE video, Spyridonos et al. [74] employed classifiers based on thirtyfour low-level image features from nine successive frames. Furthermore, a two-stage contraction detection approach was also introduced based on the SVM classifier, where the intestinal motility patterns have been encoded using morphological and textural features.

\section{RESEARCH DIRECTIONS AND CHALLENGES}

WCE has several advantages compared to traditional wiredbased endoscopy which includes less invasiveness and suitability with efficient imaging capabilities. WCE technology has progressed promptly resulting in several commercial video analysis techniques. WCE technology has progressed promptly resulting in several commercial video analysis techniques. The WCE video accessibility made a substantial influence on the medical imaging community. The passive WCE technology can be considered the first generation as it was characterized by a relatively low frame rate, low image resolution and limited battery life due to the long reviewing time. In contrast, the second generation WCE devices include electromagnetic (EM) field impulsion with remote power source that allow higher frame rates and higher resolutions with extra functions including drug delivery, biopsy, and telemetry measurement abilities. However, the WCE procedure is still slow due to the low frame rate and the long reviewing time for the physician to review and report the patient's videos. Adaptive reviewing speed control of the WCE video is a promising research area to consider the video summarization and the possible automated tools for reporting the WCE video.

These challenges led to develop new WCE video analysis procedures that used color and texture features. Different illumination correction methods such as those reported in [75] can be used as a preprocessing step before any further detection, features extraction or classification processes in the WCE images/ videos. Several classifiers can be tested using both feature sets [20]; however, most of the studies reported the superiority of the SVM classifier. Providing adequate annotated training data during the classification process is considered one of the foremost WCE research challenges. Novel computational techniques are required to support the clinical experience by developing generic approaches for image feature extraction for accurate lesions or bleeding detection. The development of new software is considered one of the procedural challenges for VCE, which requires the expansion of increased sensitivity approaches to very small size abnormalities. In addition, it is indispensable to implement detecting methods for a wide spectrum of pathologies as well as methods to diminish the review time with preserving the clinical significant information to avoid missing the detection of abnormalities. Several studies proposed discarding the redundant, uninformative or an unrepresentative large number of video frames from the WCE videos [76]. Presently, lossy compression procedures, including MPEG compression are desired because of the increased storage compression rates; while using developed lossless compression procedures in the future can achieve reduced error rates. Thus, another research direction is to preserve the quality of image/video compression for further efficient WCE video analysis.

Almost all WCE video processing researchers to date have been dedicated to the early capsules nominated for small intestine investigation. The modern technologies provide more exhaustive colon and oesophagus investigations, which requires developing advanced computer vision techniques. In addition, there are several complications that face the WCE video processing researchers, such as the capsule video data nature is mainly troublesome during the detection of the abnormality or bleeding, where usually there are only few significant abnormal cases in the video that contains about thousands of images. Accordingly, it is challenging to provide sufficient trained models from an even large number of video due to the less number of the abnormality cases in the same video, as well as due to the lack of public WCE video databases, which are essential for evaluating the performance of the video processing algorithms. The most common limitations of the WCE technology are camera's battery life, capsule size, tracking issues, image quality, existence of artifacts that affects image quality, motionbased problems along with challenges of incomplete WCE investigation and incomplete diagnostic and healing abilities [77]. From the preceding reported studies, some future directions can be suggested as follows. 
- Considering the different GI tract tissue types to explore new superior video features using context features, such as the capsule location in the 3directional view, temperature and the $\mathrm{pH}$ factor.

- Exploring the different abnormalities for efficient bleeding detection using WCE.

- Developing new computer vision research tools to detect other pathological events.

- Using an adaptive framework integrating both region/neighborhood and pixel based classifiers to detect huge number of abnormalities, which are characterized by "reddish" color.

- Adapting the WCE video play rate is another computer vision research domain. Moreover, video viewing time reduction is a challenging issue.

- Designing intelligent systems for accurate lesion recognition, abnormality localization and, potentially interactive data transmission. Such intelligent systems can empower therapeutic and actuation abilities leading to the next WCE generation. Generally, it is established that WCE systems development is a growing and active research area that requires further exploration based on advancements in video analysis. New image/ video processing stages can be developed based on efficient segmentation methods that proved their efficiency in other medical segmentation and detection problems, such as the work done in [7880].

- Using cloud computing and big data technologies as essential tools with the increased WCE data volume for effective data management. video processing can be used during the actual localization of the WCE in the body [9].

- Developing a WCE that avoids complication of using the video capsule endoscopy with Crohn's disease patients is recommended. However, video capsule endoscopy for imagining small bowel is considered as a safe routine. Capsule retention is the dominant complication of the video capsule endoscopy which causes life-threatening bowel block. Toth et al. [81] studied a capsule retention case in a colonic stent with patients suffer from Crohn's disease, where a video capsule endoscopy was used for disease's activity assessment. Furthermore, a surgery was conducted leading to patient's recovery. This work highlighted the risk of examining patients with gastrointestinal stents suffering from Crohn's disease using capsule endoscopy.

- Improving the WCE functionality and implementation to solve support biopsy, where the system of WCE is completely diagnostic and is not used in biopsy or treatment of any conditions.

\section{CONCLUSIONS}

WCE has the potential to become the prominent screening device for the entire GI tract. Computational approaches can be realized in software to enrich the diagnostic procedure for efficient and high diagnostic accuracy of the WCE screening. Recent development in the endoscopic bleeding detection procedures establishes numerous image processing methods realizing high efficiency rates. Several studies applied multiple cutting-edge image processing procedures. Most of these studies employed the images' color features that powerfully recognize the bleeding existence by the nature of the characteristic bleedings' appearance. A new research direction is to develop application recognition techniques for the initial bleeding level based on the basic features of the bleeding, rather than directly detect the bleeding presence. A shift to expert systems and cognitive methods are considered to solve such objective. Furthermore, the analysis of the visual features of the physicians can be considered as a new research trend for bleeding detection.

Computerized methodologies for detecting GI bleeding, lesions, abnormalities and the different parts of the GI tract in WCE image videos have attracted much attention with the aim to relieve physicians' workload. Low resolution, poor contrast, low frame rate and long reviewing time are considered the most challenging issues in the WCE technology. In order to resolve these limitations, various CAD systems have been developed based on WCE video analysis and computer vision along with the exploration of novel techniques and clinical pathways.

State-of-the-art procedures include potential clinically feasible intelligent software schemes that are proficient for decreasing the diagnostic effort, time and diagnosis errors while localizing abnormalities and evaluating intestinal motility. Such software should provide boosted video quality to improve the content perception.

\section{Acknowledgements:}

Ethical approval: This article does not contain any studies with human participants or animals performed by any of the authors.

Funding: We are the authors confirm no funding obtained. Conflict of Interest: We are the authors confirm that no conflict of interest.

\section{REFERENCES}

[1] Xin W, Yan G, Wang W. Study of a wireless power transmission system for an active capsule endoscope. The international journal of medical robotics and computer assisted surgery. 2010 Mar; 6(1):113-22.

[2] Iddan G, Meron G, Glukhovsky A, Swain P. Wireless capsule endoscopy. Nature. 2000 May;405(6785):417.

[3] Wang A, Banerjee S, Barth BA, Bhat YM, Chauhan S, Gottlieb KT, Konda V, Maple JT, Murad F, Pfau PR, Pleskow DK. Wireless capsule endoscopy. Gastrointestinal endoscopy. 2013 Dec 1;78(6):805-15. [4] Triester SL, Leighton JA, Leontiadis GI, Fleischer DE, Hara AK, Heigh RI, Shiff AD, Sharma VK. A meta-analysis of the yield of capsule endoscopy compared to other diagnostic modalities in patients with obscure gastrointestinal bleeding. The American journal of gastroenterology. 2005 Nov;100(11):2407.

[5] Mylonaki M, Fritscher-Ravens A, Swain P. Wireless capsule endoscopy: a comparison with push enteroscopy in patients with gastroscopy and colonoscopy negative gastrointestinal bleeding. Gut. 2003 Aug 1;52(8):1122-6.

[6] Moglia A, Menciassi A, Dario P. Recent patents on wireless capsule endoscopy. Recent Patents on Biomedical Engineering. 2008 Jan 1;1(1):24-33.

[7] Liedlgruber M, Uhl A. Computer-aided decision support systems for endoscopy in the gastrointestinal tract: a review. 
IEEE reviews in biomedical engineering. 2011 Nov 9;4:7388.

[8] Eliakim R, Fischer D, Suissa A, Yassin K, Katz D, Guttman N, Migdal M. Wireless capsule video endoscopy is a superior diagnostic tool in comparison to barium followthrough and computerized tomography in patients with suspected Crohn's disease. European journal of gastroenterology \& hepatology. 2003 Apr 1;15(4):363-7.

[9] Dey N, Ashour AS, Shi F, Sherratt RS. Wireless capsule gastrointestinal endoscopy: Direction-of-arrival estimation based localization survey. IEEE reviews in biomedical engineering. 2017 Apr 25;10:2-11.

[10] Ciuti G, Caliò R, Camboni D, Neri L, Bianchi F, Arezzo A, Koulaouzidis A, Schostek S, Stoyanov D, Oddo CM, Magnani B. Frontiers of robotic endoscopic capsules: a review. Journal of Micro-Bio Robotics. 2016 Jun 1;11(14):1-8.

[11] Koulaouzidis A, Dabos KJ. Looking forwards: not necessarily the best in capsule endoscopy?. Annals of Gastroenterology: Quarterly Publication of the Hellenic Society of Gastroenterology. 2013;26(4):365.

[12] Zhou M. On the accuracy of wireless capsule endoscope RF and visual localization.Doctoral thesis, Worcester Polytechnic Institute, USA, 2015.

[13] Iakovidis DK, Maroulis DE, Karkanis SA. An intelligent system for automatic detection of gastrointestinal adenomas in video endoscopy. Computers in Biology and Medicine. 2006 Oct 1;36(10):1084-103.

[14] Szeliski R. Computer vision: algorithms and applications. Springer Science \& Business Media; 2010 Sep 30 .

[15] Ojala T, Pietikäinen M, Mäenpää T. Multiresolution gray-scale and rotation invariant texture classification with local binary patterns. IEEE Transactions on Pattern Analysis \& Machine Intelligence. 2002 Jul 1(7):971-87.

[16] Lowe DG. Distinctive image features from scaleinvariant keypoints. International journal of computer vision. 2004 Nov 1;60(2):91-110.

[17] Dalal N, Triggs B. Histograms of oriented gradients for human detection. Proc. CVPR, San Diego, CA, USA, 2005, vol. 1, pp. 886-893.

[18] Berens J, Finlayson GD, Qiu G. Image indexing using compressed colour histograms. IEE Proceedings-Vision, Image and Signal Processing. 2000 Aug 1;147(4):349-55.

[19] Berens J, Fisher M. Wireless capsule endoscopy color video segmentation. IEEE Transactions on Medical Imaging. 2008 May 23;27(12):1769-81.

[20] Gong Y, Chuan CH, Xiaoyi G. Image indexing and retrieval based on color histograms. Multimedia Tools and Applications. 1996 Mar 1;2(2):133-56.

[21] Manjunath BS, Ohm JR, Vasudevan VV, Yamada A. Color and texture descriptors. IEEE Transactions on circuits and systems for video technology. 2001 Jun;11(6):703-15.

[22] Seguí S, Drozdzal M, Pascual G, Radeva P, Malagelada C, Azpiroz F, Vitria J. Deep learning features for wireless capsule endoscopy analysis. InIberoamerican Congress on Pattern Recognition 2016 Nov 8 (pp. 326-333). Springer, Cham.

[23] Connah D, Finlayson GD. Using local binary pattern operators for colour constant image indexing. InConference on Colour in Graphics, Imaging, and Vision 2006 Jan 1 (Vol. 2006, No. 1, pp. 60-64). Society for Imaging Science and Technology.
[24] Ojala T, Pietikäinen M, Mäenpää T. Gray scale and rotation invariant texture classification with local binary patterns. InEuropean Conference on Computer Vision 2000 Jun 26 (pp. 404-420). Springer, Berlin, Heidelberg.

[25] Lee J, Oh J, Shah SK, Yuan X, Tang SJ. Automatic classification of digestive organs in wireless capsule endoscopy videos. InProceedings of the 2007 ACM symposium on Applied computing 2007 Mar 11 (pp. 10411045). ACM.

[26] Fisher L, Krinsky ML, Anderson MA, Appalaneni V, Banerjee S, Ben-Menachem T, Cash BD, Decker GA, Fanelli RD, Friis C, Fukami N. The role of endoscopy in the management of obscure GI bleeding. Gastrointestinal endoscopy. 2010 Sep 1;72(3):471-9. [27] Dey N, Ashour A, editors. Classification and clustering in biomedical signal processing. Hershey: IGI global; 2016 Apr 7.

[28] AlShahrani AM, Al-Abadi MA, Al-Malki AS, Ashour AS, Dey N. Automated system for crops recognition and classification. InComputer Vision: Concepts, Methodologies, Tools, and Applications 2018 (pp. 1208-1223). IGI Global. [29] Saba L, Dey N, Ashour AS, Samanta S, Nath SS, Chakraborty S, Sanches J, Kumar D, Marinho R, Suri JS. Automated stratification of liver disease in ultrasound: An online accurate feature classification paradigm. Computer methods and programs in biomedicine. 2016 Jul 1;130:11834.

[30] Ahmed SS, Dey N, Ashour AS, Sifaki-Pistolla D, Bălas-Timar D, Balas VE, Tavares JM. Effect of fuzzy partitioning in Crohn's disease classification: a neuro-fuzzybased approach. Medical \& biological engineering \& computing. 2017 Jan 1;55(1):101-15. [31] Virmani J, Dey N, Kumar V. PCA-PNN and PCA-SVM based CAD systems for breast density classification. InApplications of intelligent optimization in biology and medicine 2016 (pp. 159-180). Springer, Cham.

[32] Wang P, Krishnan SM, Kugean C, Tjoa MP. Classification of endoscopic images based on texture and neural network. In2001 Conference Proceedings of the 23rd Annual International Conference of the IEEE Engineering in Medicine and Biology Society 2001 Oct 25 (Vol. 4, pp. 3691-3695). IEEE.

[33] Figueiredo IN, Prasath S, Tsai YH, Figueiredo PN. Automatic detection and segmentation of colonic polyps in wireless capsule images. ICES REPORT. 2010 Sep 22:1036.

[34] Karargyris A, Bourbakis N. Detection of small bowel polyps and ulcers in wireless capsule endoscopy videos. IEEE transactions on BioMedical Engineering. 2011 May 16;58(10):2777-86.

[35] Zhao Q, Meng MQ. Polyp detection in wireless capsule endoscopy images using novel color texture features. In2011 9th World Congress on Intelligent Control and Automation 2011 Jun 21 (pp. 948-952). IEEE.

[36] Bourbakis N, Makrogiannis S, Kavraki D. A neural network-based detection of bleeding in sequences of WCE images. InFifth IEEE Symposium on Bioinformatics and Bioengineering (BIBE'05) 2005 Oct 19 (pp. 324-327). IEEE. [37] Jung YS, Kim YH, Lee DH, Kim JH. Active blood detection in a high resolution capsule endoscopy using color spectrum transformation. In2008 International Conference on BioMedical Engineering and Informatics 2008 May 27 (Vol. 1, pp. 859-862). IEEE.

[38] Li B, Meng MQ. Computer-based detection of bleeding and ulcer in wireless capsule endoscopy images by 
chromaticity moments. Computers in biology and medicine. 2009 Feb 1;39(2):141-7.

[39] Li B, Meng MQ. Small bowel tumor detection for wireless capsule endoscopy images using textural features and support vector machine. In2009 IEEE/RSJ International Conference on Intelligent Robots and Systems 2009 Oct 10 (pp. 498-503). IEEE.

[40] Yu L, Yuen PC, Lai J. Ulcer detection in wireless capsule endoscopy images. InProceedings of the 21st International Conference on Pattern Recognition (ICPR2012) 2012 Nov 11 (pp. 45-48). IEEE.

[41] Yuan Y, Meng MQ. Deep learning for polyp recognition in wireless capsule endoscopy images. Medical physics. 2017 Apr;44(4):1379-89.

[42] Kumar R, Zhao Q, Seshamani S, Mullin G, Hager G, Dassopoulos T. Assessment of Crohn's disease lesions in wireless capsule endoscopy images. IEEE Transactions on biomedical engineering. 2011 Oct 18;59(2):355-62.

[43] Sekuboyina AK, Devarakonda ST, Seelamantula CS. A convolutional neural network approach for abnormality detection in wireless capsule endoscopy. In2017 IEEE 14th International Symposium on Biomedical Imaging (ISBI 2017) 2017 Apr 18 (pp. 1057-1060). IEEE.

[44] Zou Y, Li L, Wang Y, Yu J, Li Y, Deng WJ. Classifying digestive organs in wireless capsule endoscopy images based on deep convolutional neural network. In2015 IEEE International Conference on Digital Signal Processing (DSP) 2015 Jul 21 (pp. 1274-1278). IEEE.

[45] Cunha JS, Coimbra M, Campos P, Soares JM. Automated topographic segmentation and transit time estimation in endoscopic capsule exams. IEEE Transactions on Medical Imaging. 2007 Dec 26;27(1):19-27.

[46] Vilarino F, Spyridonos P, DeIorio F, Vitria J, Azpiroz F, Radeva P. Intestinal motility assessment with video capsule endoscopy: automatic annotation of phasic intestinal contractions. IEEE transactions on medical imaging. 2009 May 5;29(2):246-59.

[47] Yagi Y, Vu H, Echigo T, Sagawa R, Yagi K, Shiba M, Higuchi K, Arakawa T. A diagnosis support system for capsule endoscopy. Inflammopharmacology. 2007 Apr $1 ; 15(2): 78-83$.

[48] Szczypiński PM, Sriram RD, Sriram PV, Reddy DN. A model of deformable rings for interpretation of wireless capsule endoscopic videos. Medical Image Analysis. 2009 Apr 1;13(2):312-24.

[49] Appleyard M, Fireman Z, Glukhovsky A, Jacob H, Shreiver R, Kadirkamanathan S, Lavy A, Lewkowicz S, Scapa E, Shofti R, Swain P. A randomized trial comparing wireless capsule endoscopy with push enteroscopy for the detection of small-bowel lesions. Gastroenterology. 2000 Dec 1;119(6):1431-8.

[50] de Iorio F, Radeva P, Vitria J, Pujol O, Spyridonos P, Vilarino F. Automatic detection of intestinal juices in wireless capsule video endoscopy. In18th International Conference on Pattern Recognition (ICPR'06) 2006 Aug 20 (Vol. 4, pp. 719-722). IEEE.

[51] Bashar MK, Kitasaka T, Suenaga Y, Mekada Y, Mori $\mathrm{K}$. Automatic detection of informative frames from wireless capsule endoscopy images. Medical Image Analysis. 2010 Jun 1;14(3):449-70.

[52] Wang C, Luo Z, Liu X, Bai J, Liao G. Detection of protruding lesion in wireless capsule endoscopy videos of small intestine. InMedical Imaging 2018: Computer-Aided
Diagnosis 2018 Feb 27 (Vol. 10575, p. 1057513). International Society for Optics and Photonics.

[53] Vu H, Echigo T, Sagawa R, Yagi K, Shiba M, Higuchi $\mathrm{K}$, Arakawa T, Yagi Y. Contraction detection in small bowel from an image sequence of wireless capsule endoscopy. InInternational Conference on Medical Image Computing and Computer-Assisted Intervention 2007 Oct 29 (pp. 775783). Springer, Berlin, Heidelberg.

[54] Vu H, Echigo T, Sagawa R, Yagi K, Shiba M, Higuchi $\mathrm{K}$, Arakawa T, Yagi Y. Detection of contractions in adaptive transit time of the small bowel from wireless capsule endoscopy videos. Computers in biology and medicine. 2009 Jan 1;39(1):16-26.

[55] Malagelada C, De Iorio F, Azpiroz F, Accarino A, Segui S, Radeva P, Malagelada JR. New insight into intestinal motor function via noninvasive endoluminal image analysis. Gastroenterology. 2008 Oct 1;135(4):1155-62.

[56] Spyridonos P, Vilariño F, Vitrià J, Azpiroz F, Radeva P. Anisotropic feature extraction from endoluminal images for detection of intestinal contractions. InInternational Conference on Medical Image Computing and ComputerAssisted Intervention 2006 Oct 1 (pp. 161-168). Springer, Berlin, Heidelberg. [57] Vilariño F, Spyridonos P, Vitrià J, Malagelada C, Radeva P. Linear radial patterns characterization for automatic detection of tonic intestinal contractions. InIberoamerican Congress on Pattern Recognition 2006 Nov 14 (pp. 178-187). Springer, Berlin, Heidelberg.

[58] Drozdzal M, Seguí S, Vitrià J, Malagelada C, Azpiroz F, Radeva P. Adaptable image cuts for motility inspection using WCE. Computerized Medical Imaging and Graphics. 2013 Jan 1;37(1):72-80.

[59] Szczypinski PM, Sriram PV, Sriram RD, Reddy D. Computerized Image Analysis of Wireless Capsule Endoscopy Videos Using a Dedicated Web-like Model of Deformable Rings-A Feasibility Study. 12th United European Gastroenterology Week, Prague. 2004 Sep;36(Suppl I):A76.

[60] Okuhata H, Nakamura H, Hara S, Tsutsui H, Onoye T. Application of the real-time Retinex image enhancement for endoscopic images. In2013 35th Annual International Conference of the IEEE Engineering in Medicine and Biology Society (EMBC) 2013 Jul 3 (pp. 3407-3410). IEEE. [61] Ramaraj M, Raghavan S, Khan WA. Homomorphic filtering techniques for WCE image enhancement. In2013 IEEE International Conference on Computational Intelligence and Computing Research 2013 Dec 26 (pp. 1-5). IEEE.

[62] Li B, Meng MQ. Wireless capsule endoscopy images enhancement via adaptive contrast diffusion. Journal of Visual Communication and Image Representation. 2012 Jan 1;23(1):222-8.

[63] Gopi VP, Palanisamy P. Capsule endoscopic image denoising based on double density dual tree complex wavelet transform [J]. International Journal of Imaging and Robotics. 2013;9(1):48-60.

[64] Karargyris A, Bourbakis N. An elastic video interpolation methodology for wireless capsule endoscopy videos. In2010 IEEE International Conference on BioInformatics and BioEngineering 2010 May 31 (pp. 3843). IEEE.

[65] Häfner M, Liedlgruber M, Uhl A. POCS-based superresolution for HD endoscopy video frames. InProceedings of 
the 26th IEEE International Symposium on Computer-Based Medical Systems 2013 Jun 20 (pp. 185-190). IEEE.

[66] Hai V, Echigo T, Sagawa R, Yagi K, Shiba M, Higuchi $\mathrm{K}$, Arakawa T, Yagi Y. Adaptive control of video display for diagnostic assistance by analysis of capsule endoscopic images. In18th International Conference on Pattern Recognition (ICPR'06) 2006 Aug 20 (Vol. 3, pp. 980-983). IEEE.

[67] Lewis BS, Swain P. Capsule endoscopy in the evaluation of patients with suspected small intestinal bleeding: results of a pilot study. Gastrointestinal endoscopy. 2002 Sep 1;56(3):349-53.

[68] Chu X, Poh CK, Li L, Chan KL, Yan S, Shen W, Htwe TM, Liu J, Lim JH, Ong EH, Ho KY. Epitomized summarization of wireless capsule endoscopic videos for efficient visualization. InInternational Conference on Medical Image Computing and Computer-Assisted Intervention 2010 Sep 20 (pp. 522-529). Springer, Berlin, Heidelberg.

[69] Szeliski R. Image alignment and stitching: A tutorial. Foundations and Trends $₫$ in Computer Graphics and Vision. 2007 Jan 2;2(1):1-04.

[70] Jia X, Meng MQ. A deep convolutional neural network for bleeding detection in wireless capsule endoscopy images. In2016 38th Annual International Conference of the IEEE Engineering in Medicine and Biology Society (EMBC) 2016 Aug 16 (pp. 639-642). IEEE.

[71] Li B, Meng MQ. Texture analysis for ulcer detection in capsule endoscopy images. Image and Vision computing. 2009 Aug 3;27(9):1336-42.

[72] Charisis VS, Katsimerou C, Hadjileontiadis LJ, Liatsos CN, Sergiadis GD. Computer-aided capsule endoscopy images evaluation based on color rotation and texture features: An educational tool to physicians. InProceedings of the 26th IEEE International Symposium On Computer-Based Medical Systems 2013 Jun 20 (pp. 203-208). IEEE.

[73] Boulougoura M, Wadge E, Kodogiannis V, Chowdrey HS. Intelligent systems for computer-assisted clinical endoscopic image analysis. InSecond International Conference on Biomedical Engineering 2004. Acta Press.

[74] Spyridonos P, Vilariño F, Vitria J, Radeva P. Identification of intestinal motility events of capsule endoscopy video analysis. InInternational Conference on Advanced Concepts for Intelligent Vision Systems 2005 Sep 20 (pp. 531-537). Springer, Berlin, Heidelberg.

[75] Dey N. Uneven illumination correction of digital images: A survey of the state-of-the-art. Optik. 2019 Apr 1;183:483-95.

[76] Yuan Y, Meng MQ. Hierarchical key frames extraction for WCE video. In2013 IEEE International Conference on Mechatronics and Automation 2013 Aug 4 (pp. 225-229). IEEE.

[77] Kwack WG, Lim YJ. Current status and research into overcoming limitations of capsule endoscopy. Clinical endoscopy. 2016 Jan;49(1):8.

[78] Razmjooy N, Mousavi BS, Soleymani F. A hybrid neural network Imperialist Competitive Algorithm for skin color segmentation. Mathematical and Computer Modelling. 2013 Feb 1;57(3-4):848-56.

[79] Razmjooy N, Sheykhahmad FR, Ghadimi N. A hybrid neural network-world cup optimization algorithm for melanoma detection. Open Medicine. 2018 Jan 1;13(1):9-16. [80] Mirjalili S, Hashim SZ, Sardroudi HM. Training feedforward neural networks using hybrid particle swarm optimization and gravitational search algorithm. Applied Mathematics and Computation. 2012 Jul 15;218(22):1112537.

[81] Toth E, Marthinsen L, Bergström M, Park PO, Månsson P, Nemeth A, Johansson GW, Thorlacius H. Colonic obstruction caused by video capsule entrapment in a metal stent. Annals of translational medicine. 2017 May;5(9). 